\title{
Bacterial Sex Pheromone-Induced Plasmid Transfer
}

\author{
Don B. Clewell \\ Departments of Biologic/Materials Sciences \\ and Microbiology/lmmunology \\ The University of Michigan \\ Ann Arbor, Michigan 48109
}

Multiple antibiotic resistance is a major clinical problem. Plasmids and conjugative transposons play a key role in the acquisition of not only drug resistance but certain virulence traits (e.g., hemolysin determinants). How these plasmids and transposons move is of increasing interest, because recovery from certain diseases depends completely on antibiotic chemotherapy. For this reason, for example, infective endocarditis is one of the most feared diseases caused by Enterococcus (formerly Streptococcus) faecalis, a gram-positive bacterium commonly found in the human intestine and frequently involved in urinary tract and other infections (Clewell, 1981, 1990).

An interesting feature thus far unique to the enterococci is the role played by sex pheromones in the transfer of a number of different conjugative plasmids. Plasmid-free (recipient) strains of $E$. faecalis secrete a family of heatstable, protease-sensitive pheromones with specificities for donors carrying various conjugative plasmids (Dunny et al., 1978, 1979). Bacteria carrying a particular plasmid respond by synthesizing a proteinaceous adhesin that facilitates formation of mating aggregates with nearby recipients. Chemotaxis does not appear to be involved; donors and recipients seem to make contact by random collisions. Mixtures of donors and recipients in liquid culture were observed to "clump out" of solution after 2-4 hr. In fact, a culture filtrate from a plasmid-free strain is sufficient to induce self-aggregation of a suspension of donors only.

Pheromone was initially isolated by virtue of its activities as both a "clumping inducing agent" and a "fertility boosting ingredient," which increases the transfer frequency of a plasmid by as much as $10^{5}$ - to $10^{6}$-fold. The induced surface material on donors is referred to as aggregation substance, and its receptor on the recipient has been designated binding substance. Binding substance is also present on the donor surface (i.e., the reason for inducible self-clumping) and may correspond at least in part to lipoteichoic acid, which in microgram amounts can block clumping (Ehrenfeld et al., 1986).

Once a copy of the plasmid has been acquired, the recipient shuts down production of that pheromone but continues to secrete pheromones specific for other plasmids. The four sex pheromones that have been characterized (Suzuki et al., 1984; see Clewell, 1990) are hydrophobic peptides of 7 or 8 amino acids. Each pheromone results in mating responses by cells harboring a particular plasmid. There is evidence that induction can result from the interaction of a donor with relatively few molecules of pheromone (Mori et al., 1988).

Interestingly, each plasmid in turn encodes a peptide,
Minireview

which is secreted and acts as a specific competitive inhibitor of the corresponding pheromone (Mori et al., 1986). These peptides could block self-induction that might result from incomplete pheromone shutdown in the donor. They could also desensitize donors to pheromone that is produced by recipients too distant to be encountered by random collision. When equal numbers of donors and recipients are present, pheromone generally outcompetes the inhibitor.

The best-studied pheromone-related plasmid systems are PAD1 and pCF10. pAD1, a hemolysin/bacteriocin plasmid, confers a response to the pheromone CAD1; pCF10 determines tetracycline resistance, which is carried by the transposon Tn925.

\section{The pAD1 System}

pAD1 was originally identified in the $E$. faecalis clinical isolate DS16, which was resistant to multiple antibiotics (see Clewell, 1990). At least half of pAD1 is devoted to functions related to conjugation (Figure 1). A contiguous segment from $\sim 17.5 \mathrm{~kb}$ counterclockwise to $\sim 45 \mathrm{~kb}$ contains determinants (transcribed counterclockwise) inducible by the pheromone CAD1 (Ehrenfeld and Clewell, 1987; Pontius and Clewell, 1991; Clewell, 1993). This region includes the determinants for aggregation substance (asa 1) and surface exclusion (sea1) as well as determinants involved in stabilization of newly formed aggregates (region G) and DNA transfer (region H). Transposon (Tn 917 lac) insertions in region I, which reveal transcriptional inducibility, exhibit no detectable phenotype. It may be involved in a function that is not easily measured under laboratory mating conditions, such as dissociation after DNA transfer.

Comparison of the sequences of Asa1 and Sea1 with the corresponding products of pCF10 (Asc10 and Sec10, respectively) shows extensive homology in both cases (see Dunny, 1990; Clewell, 1993). Furthermore, the pCF10 genes that determine aggregation substance and surface exclusion are adjacent and in the same orientation, as they are on pAD1 (Dunny, 1990; Kao et al., 1991). sea1 and asa1 have strong similarities to a number of additional enterococcal plasmids exhibiting pheromone responses. A notable exception is pAM373, which is also unique in that an activity resembling its corresponding pheromone (CAM373) is produced by essentially all strains of Staphylococcus aureus.

The aggregation substance asa1 gene encodes a 142 kd signal peptide-containing protein (Galli et al., 1990). The C-terminal region contains a proline-rich sequence followed by a membrane anchor with significant similarity to surface proteins of other gram-positive bacteria (e.g., M protein of Streptococcus pyogenes). The predominant protein detected on Western blots is $74 \mathrm{kd}$, which represents the $\mathrm{N}$-terminal half of Asa1. Immunoelectron microscopy using an antiserum against the $74 \mathrm{kd}$ protein detects microfibrillar surface structures ("hairs"). Interestingly, Asa1 contains two Arg-Gly-Asp motifs, which are recep- 

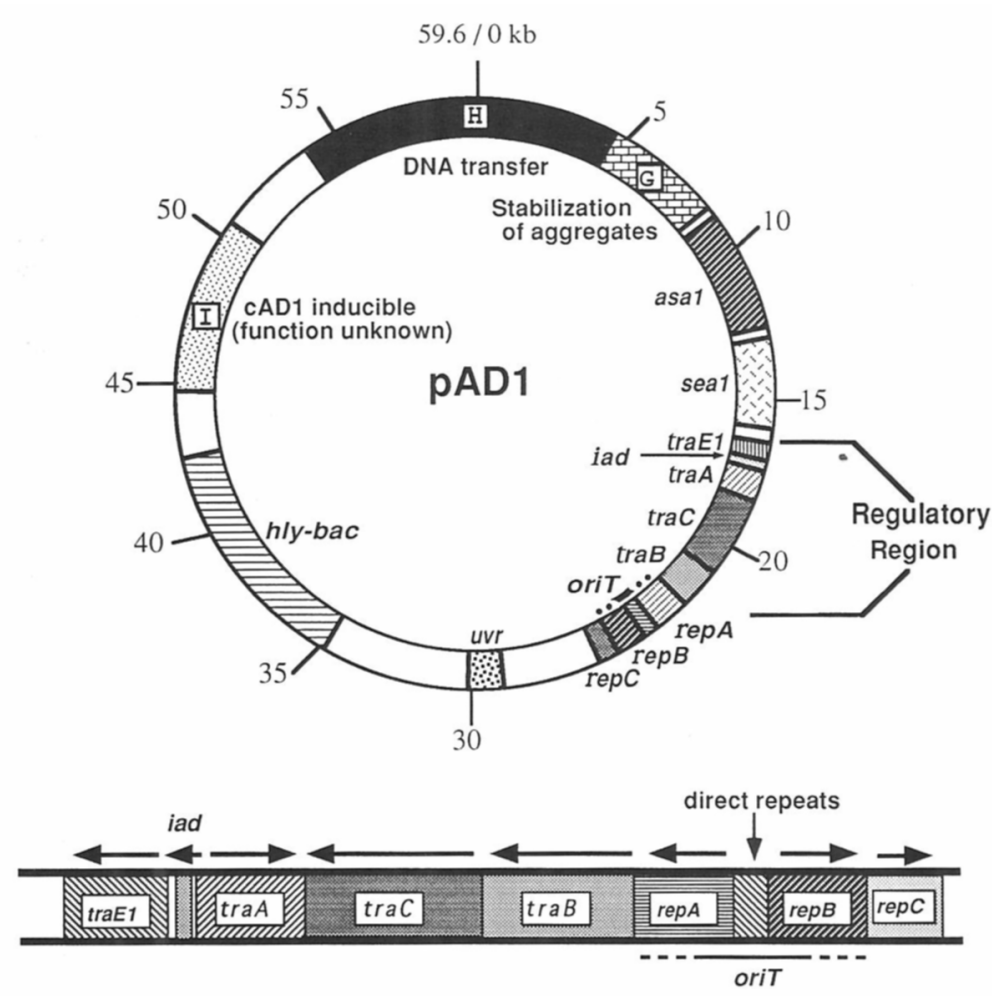

Figure 1. Map of pAD1

Expanded region contains genes related to regulation of the pheromone response. Arrows indicate the direction of transcription. tors for integrins on the surface of eukaryotic cells. Consistent with this, E. faecalis cells containing PAD1 adhere to cultured pig renal tubular cells to a greater extent than do plasmid-free cells (Kreft et al., 1992).

Surface exclusion minimizes exchange of plasmids between donors. Uninduced donors are good recipients for uptake of homologous plasmid. DNA; induction by the relevant pheromone reduces recipient potential by $10-$ to 100 -fold. The phenomenon, however, is pheromone specific. For example, induction with the pheromone CCF10 results in exclusion of incoming pCF10 but not pAD1. Like Asa1, the product of the surface exclusion determinant sea 1 has a signal sequence and a typical membrane anchor region. Sea1 exhibits some homology with portions of $M$ proteins from $S$. pyogenes as well as structural proteins of eukaryotes, such as myosin or laminin (Weidlich et al., 1992). A monoclonal antibody preparation against Sec10 interferes with exclusion of pCF10 (Dunny, 1990). The basis of surface exclusion is not known. However, since surface exclusion proteins are extracellular, they may act by blocking formation of "fusion points" for DNA transfer between aggregated cells.

\section{Regulation of the Response}

Genes that regulate the pheromone response are adjacent to the surface exclusion determinant (Figure 1; Weaver and Clewell, 1988; Clewell and Weaver, 1989; Clewell, 1993). The product of traE1 is believed to be a key positive regulator, since related mutations (transposon insertions) abolish the expression of most if not all the downstream structural genes. TraE1 probably acts at multiple sites, since transposon insertions downstream do not exhibit polarity.
In contrast, a segment of pCF10 DNA containing $\operatorname{prg} X, Q, R, S, T$ exhibits positive control in a cis-acting, orientation-dependent manner that can occur at distances greater than $10 \mathrm{~kb}$ from its target prgB, which determines aggregation substance (Chung and Dunny, 1992). Table 1 lists determinants involved in various functions for both PAD1 and pCF10. A model involving tracking from upstream of prgR has been proposed. The significant difference in the mechanisms of positive regulation of PCF10 and PAD1 is reflected in the lack of apparent homology in the regions of each plasmid that are involved in positive regulation.

In the case of PAD1, the pheromone-inhibitor peptide IAD1 is encoded in the iad determinant upstream of traE1. The peptide is synthesized as a 22 amino acid precursor, in which the last 8 residues correspond to iAD1. In pCF10, prgQ is thought to encode the pheromone-inhibitor peptide.

Upstream of iad is a determinant traA, which encodes

\begin{tabular}{lll}
\hline \multicolumn{3}{l}{ Table 1. pAD1 and pCF10 Determinants } \\
\hline \multicolumn{3}{l}{ Determinant } \\
\cline { 2 - 3 } Function/Role & pAD1 & pCF10 \\
\hline Aggregation substance & asa1 & prgB (asc10) \\
Surface exclusion & sea1 & prgA (sec10) \\
Inhibitor peptide & lad & prgQ \\
Positive regulation & traE1 & prgR,S,T,X \\
Negative regulation & traA & $?$ \\
Pheromone sensing & traC & prgZ \\
Pheromone shutdown & traB & prgY? \\
\hline
\end{tabular}

a. Dunny, personal communication (orgz has extensive homology with traC, as does prgY with traB.) 


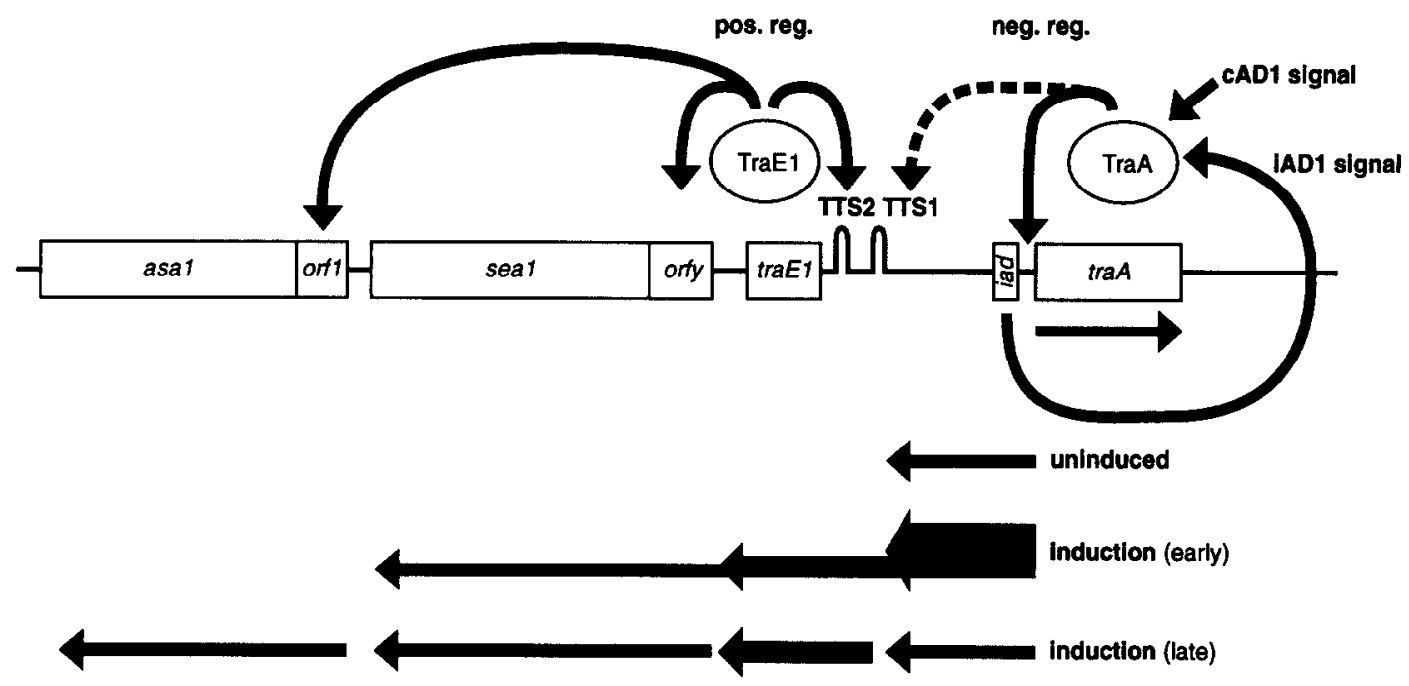

Figure 2. Model for the Regulation of the pAD1-Encoded Pheromone Response

The arrows below the diagram reflect the level (qualitatively) and direction of transcription of various regions. The functions of orf1 and orfy are not known, although sea1 is probably cotranscribed with orfy. TTS, transcription termination site. From Tanimoto and Clewell (1993); reproduced with the permission of the American Society of Microbiology.

a key negative regulator (Ike and Clewell, 1984; Weaver and Clewell, 1988). Mutations in traA result in constitutive expression of essentially all structural genes related to the mating response, because TraA normally represses expression of TraE1. In contrast with all the other genes in the regulatory region, traA is oriented clockwise (Figure 1). It is expressed constitutively at a low level. Certain mutations involving insertions near the $3^{\prime}$ end of tra $A$ exhibit only a low level of derepression. However, since these mutants are insensitive to further induction by pheromone, they also reflect loss of TraA's ability to interact directly or indirectly (i.e., via signal transduction) with the pheromone CAD1. There is also evidence for at least one determinant involved in negative regulation of pCF10 structural genes. No apparent local homology has been found between TraA and key signal transduction proteins of two-component regulatory systems.

Figure 2 represents a working hypothesis for how the pAD1 pheromone response is regulated (Galli et al., 1992; Pontius and Clewell, 1992; Tanimoto and Clewell, 1993). At the heart of the system is control of the level of transcriptional readthough from iad into traE1. There is significant transcription in the absence of pheromone upstream of transcription termination site 1 , which is consistent with the presence of extracellular iAD1. On the other hand, immediately downstream of transcription termination site 1 , transcription is undetectable but is highly induced after exposure of cells to pheromone. Readthrough occurs into traE1 and even into sea 1 for $\sim 20 \mathrm{~min}$ (designated "early"), after which there is a shift to synthesis of transcripts corresponding individually to traE1 and sea1.

At this point (designated "late"), transcripts for asa1 are evident. The data have been interpreted as indicating that, once enough TraE1 has been produced as a result of transcriptional readthough, TraE1 activates not only the promoters of asa 1 and sea1 but its own promoter as well. It would seem that TraA must play some role in the transcription termination at site 1. However, DNA binding studies thus far have detected binding of TraA only between iad and tra $A$, indicating some degree of negative regulation of lad and perhaps itself. This is consistent with the observed increased synthesis of /ad-related RNA during early induction; increases in iAD1 appearance (which could be measured only indirectly) seemed to be only about 2-fold, however. It is noteworthy that the nucleotide sequence between iad and transcription termination site 1 reveals a large amount of potential secondary structure. It is conceivable that TraA acts together with other factors to facllitate regulation (i.e., by modulating transcriptional readthough) at transcription termination site 1.

TraC, a $61 \mathrm{kd}$ protein with an $\mathrm{N}$-terminal signal sequence (see Clewell, 1993), exhibits a high degree of homology with oligopeptide-binding proteins from Escherichia coli, Salmonella typhimurium, and Bacillus subtilis. Insertions in this region result in a decreased sensitivity to the pheromone CAD1 and are characterized by a 4- to 8-fold elevation of the inhibitor IAD1 in culture filtrates. It is likely that TraC is a CAD1-binding protein and/or receptor, and the increased levels of free iAD1 are believed to result from a failure to associate with TraC.

The nucleotide sequence of traB predicts a $44 \mathrm{kd}$ protein (see Clewell, 1993). TraB is believed to be involved in shutdown of endogenous CAD1. However, traB mutants still exhibit lower than plasmid-free levels of pheromone, implying that other factors also participate in pheromone shutdown. It is interesting that pheromone production by plasmid-fre日 cells is significantly affected by aeration, as cultures grown with vigorous shaking secrete only $5 \%-7 \%$ as much pheromone as cultures grown without shaking. When the plasmid PAD1 is present, the shutdown mechanism must therefore be able to compensate for whatever level is being produced. 
Adjacent to traB there are several open reading frames (see Figure 1) as well as a series of direct repeats (Weaver et al., 1993). There is evidence that the region consisting of repA-direct repeats-repB contains the origin of transfer (oriT). When this region is carried by a different plasmid vector, the chimera is readily mobilized by a conjugative derivative of pAD1 (Clewell, 1993). A specific nicking site precisely defining the origin, however, has not yet been determined. There is also good evidence that the segment consisting of repA-direct repeats-repB-repC is involved in plasmid replication and maintenance (Weaver et al., 1993).

An interesting feature of the pAD1 conjugation system is its ability to undergo phase variation (Pontius and Clewell, 1991). At a frequency of about $10^{-4}$ to $10^{-3}$, the entire system becomes spontaneously switched on, leading to a constitutive derepression of all the structural genes associated with conjugative transfer. Reversal occurs at a similar frequency. Efforts to relate the phase variation to structural changes in the DNA involving traA or the iad-traE1 region have been unsuccessful.

\section{Concluding Remarks}

Plasmids in E. faecalis conferring responses to sex pheromones are ubiquitous and can transfer at frequencies approaching $100 \%$ under suitable conditions. There are also conjugative plasmids in enterococci that do not make use of pheromones. In the laboratory these plasmids generally do not transfer in broth but rather on solid surfaces. However, such plasmids may transfer in broth readily, if they are present in a donor together with a pheromoneresponding plasmid. Thus, plasmids that confer a pheromone response greatly enhance the transfer of other elements. The pheromone-responding plasmids have thus far been reported to transfer only among enterococci. In contrast, certain conjugative plasmids that do not independently confer a pheromone response (e.g., PAM $\beta 1$ or pIP501) exhibit a broad host range among gram-positive species (Clewell, 1990).

Plasmid-free strains of $E$. faecalis secrete at least five and possibly many more sex pheromones. It might, therefore, be expected that some strains would have readily acquired multiple pheromone-responding plasmids. Indeed, at least two strains have been found to harbor simultaneously three plasmids, each conferring a response to a different pheromone. The production of multiple pheromones by plasmid-free strains raises the interesting question as to how these peptides evolved as important participants in the mating process. The peptides originally may have had, or continue to have, other functions; various plasmids may have simply evolved to take advantage of them as mating signals.

In this regard, it is noteworthy that certain peptide pheromones/inhibitors have been found to exhibit potent neutrophil chemotaxis activities (Sannomiya et al., 1990; Ember and Hugli, 1989). Therefore, some of these substances might have some influence on the course of a clinical infection. Since pheromones and their corresponding plasmid-encoded inhibitor peptides compete for a bacterial receptor, they might also compete for receptors on neutrophils. Thus, certain peptides might serve to block neutro- phil chemotaxis, making them virulence factors. In the case of the CAD1-iAD1 system, the inhibitor iAD1 induces neutrophil chemotaxis, whereas the pheromone (CAD1) does not. It has not been shown, however, whether CAD1 actually blocks the activity of iAD1 or other chemotaxis inducers. Additional roles for the enterococcal peptides might help explain why $E$. faecalis strains are generally not full of the many plasmids that they are constantly attracting. While under certain conditions a particular plasmid may be picked up, subsequent environmental conditions might provide selection for production of the pheromone (via curing of the plasmid) for survival purposes.

\section{References}

Chung, J. W., and Dunny, G. M. (1992). Proc. Natl. Acad. Sci. USA 89, 9020-9024.

Clewell, D. B. (1981). Microblol. Rev. 45, 409-436.

Clewell, D. B. (1990). Eur. J. Clin. Microbiol. Infect. Dis. 9, 90-102. Clewell, D. B. (1993). In Bacterial Conjugation, D. B. Clewell, ed. (New York: Plenum Press), in press.

Clewell, D. B., and Weaver, K. E. (1989). Plasmid 21, 175-184.

Dunny, G. M. (1990). Mol. Microbiol. 4, 689-696.

Dunny, G. M., Brown, B. L., and Clewell, D. B. (1978). Proc. Natl. Acad. Sci. USA 75, 3479-3483.

Dunny, G. M., Craig, R. A., Carron, R. L., and Clewell, D. B. (1979) Plasmid 2, 454-465.

Ehrenfeld, E. E., and Clewell, D. B. (1987). J. Bacteriol. 169, 34733481.

Ehrenfeld, E. E., Kessler, R. E., and Clewell, D. B. (1986). J. Bacteriol. 168, 6-12.

Ember, J. A., and Hugli, T. E. (1989). Am. J. Pathol. 134, 797-805. Galli, D., Lottspeich, F., and Wirth, R. (1990). Mol. Microbiol. 4, 895904.

Galli, D., Friesenegger, A., and Wirth, R. (1992). Mol. Microbiol. 6, 1297-1308.

Ike, Y., and Clewell, D. B. (1984). J. Bacteriol. 158, 777-783.

Kao, S., Olmsted, S. B., Viksnins, A. S., Gallo, J. C., and Dunny, G. M. (1991). J. Bacteriol. 173, 7650-7664.

Kreft, B., Marre, R., Schramm, U., and Wirth, R. (1992). Infect. Immun. 60, 25-30.

Mori, M., Isogai, A., Sakagami, Y., Fujino, M., Kitada, C., Clewell, D. B., and Suzuki, A. (1986). Agric. Biol. Chem. 50, 539-541.

Mori, M., Sakagami, Y., Ishii, Y., Isogai, A., Kitada, C., Fujino, M. Adsit, J. C., Dunny, G. M., and Suzuki, A. (1988). J. Biol. Chem. 263, 14574-14578.

Pontius, L. T., and Clewell, D. B. (1991). Plasmid 26, 172-185.

Pontius, L. T., and Clewell, D. B. (1992). J. Bacteriol. 174, 3152-3160. Sannomiya, P., Craig, R. A., Clewell, D. B., Suzukl, A., Fujlno, M., Till, G. O., and Marasco, W. A. (1990). Proc. Natl. Acad. Sci. USA 87, 66-70.

Suzuki, A., Mori, M., Sakagami, Y., Isogai, A., Fujino, M., Kitada, C., Craig, R. A., and Clewell, D. B. (1984). Science 226, 849-850.

Tanimoto, K., and Clewell, D. B. (1993). J. Bacteriol. 175, 1008-1018.

Weaver, K. E., and Clewell, D. B. (1988). J. Bacteriol. 170, 4343-4352. Weaver, K. E., Clewell, D. B., and An, F. Y. (1993). J. Bacteriol., in press.

Weidlich, G., Wirth, R., and Galli, D. (1992). Mol. Gen. Microbiol. 233, 161-168. 